\title{
The use of pollutants fate simulation models in the risk analysis
}

\author{
M. Andretta \\ Montecatini Environmental Research Centre, Edison Group, Italy
}

\begin{abstract}
In this paper, I describe the most important theoretical aspects of the polluted soil risk assessment methodologies and the relevant role played, in this kind of analysis, by the pollutant transport models. In particular, I describe a new and innovative model, based on the general framework of the so called Cellular Automata (CA), initially developed in the Esprit Project named COLOMBO (partially sponsored by EU Commission) for the bioremediation phenomena simulation [1] and successfully applied to the detailed analysis of the shape of the pollutant sources and of the contaminant fates in the risk assessment evaluation.

In particular, I present the simulation of the contaminant distribution in a complex field situation and I shortly describe the future research activities we are going to develop in the area of a strict integration between pollutant fate and transport models and Risk Analysis Methodologies.
\end{abstract}

\section{Introduction}

In the last years, risk analysis methods have been applied more frequently for polluted site assessment studies both in the North America countries and in the European ones. There are, at least, two main reasons for the increase of application of these methodologies. The first one is the necessity of a methodology for defining the technical and economical limits of soil remediation. Mainly, these limits are defined in terms of general threshold values that, in some cases, can not be reached either using the so called Best Available Technology (B.A.T.). It can depend, for example: on the characteristics of the pollutants, of the affected soil either on the extremely high cost or duration of the remedial intervention.

The second reason, particularly applicable to the so called "Hazard Ranking Systems", is related to the necessity of defining priority lists of potentially polluted sites and related environmental protection actions. The huge number of 


\section{Environmental Health Risk}

possible polluted areas in quite all industrial countries of the world and the related requirement of remedial initiatives, in many cases very expensive in nature, forces the Public Environmental Agencies or, sometimes, the site owners too, to make site assessments and to prepare priority lists on the basis of objective screening criteria [2].

\section{General characteristics of the Risk Assessment methods}

These increasing applications of risk assessment procedures produce a diversification and specialisation of different methodologies, depending on the case under considerations. The polluted soil risk assessment methodologies differ one from each other mainly on the way they model the targets and effect of the pollutants (e.g.: damages to the human life, chronicle health effects, acute health effects, damages to the ecosystems, possible genetics modifications, etc.)

Generally speaking, the Risk Analysis Systems for polluted site assessment may be divided into two classes, as reported in Figure 1.

Risk Analysis Systems

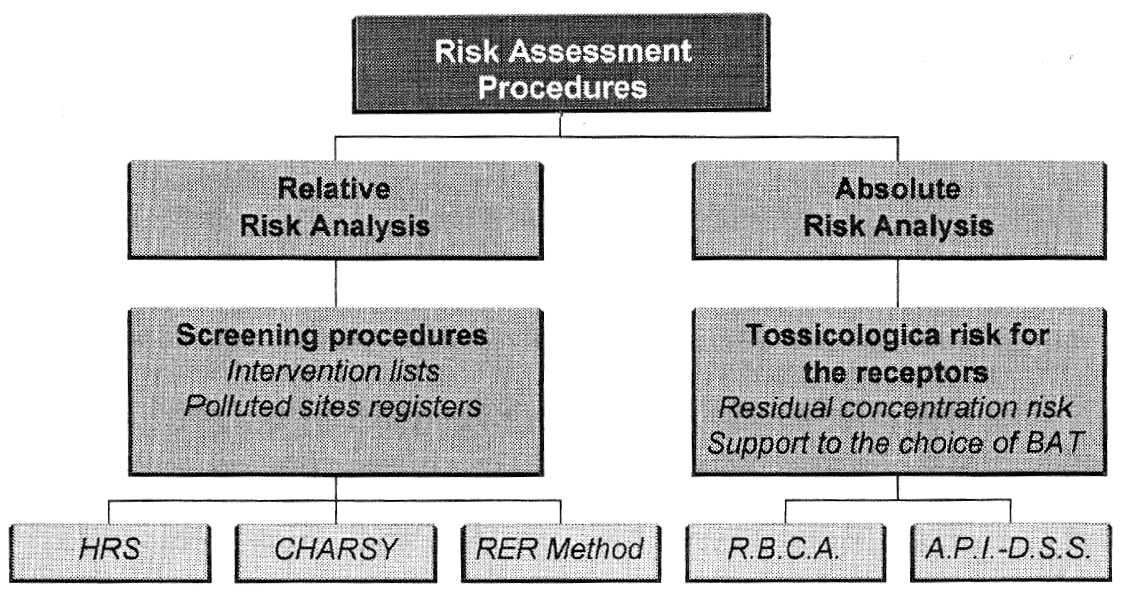

Figure 1: Classification and taxonomy of the Risk Analysis Systems

As sketched in Figure 2, all methods, both the "Absolute Risk Analysis" and the "Hazard Ranking Systems" ones, give a mathematical description of what we can define: "the main theoretical aspects of the risk analysis methods":

- the sources of contamination,

- the transport of the contaminants from the sources to the points of interest,

- the receptors, in the points of interest, and way and details of the contaminants intakes and absorption. 


\section{Sources}

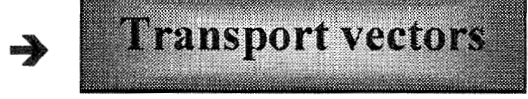

Receptors

Figure 2: The general theoretical aspects modelled by Risk Analysis Models

Anyway, there are some fundamental differences between the method of the risk assessment models and the evaluation of this theoretical framework.

Typically, the Hazard Ranking Systems produce results that can be used a ranking purpose. The scores that are obtained with these methods have not a meaning by themselves, but they have to be related with analogous results obtained with the application of the same methodology to other sides. For these reasons, these ranking systems model the three main theoretical aspects in a quite simplified way, based on scores arising from semi-empirical considerations.

If we analyse, just for example, the Hazard Ranking Systems (HRS) and its modification and updated version the so called CHARSY Method (C.r.a. Hazard Ranking System), elaborated by CRA (Centro Ricerche Ambientali Montecatini, Montecatini Environmental Research Centre), we can notice that this method considers the above mentioned three different categories of relevant factors (sources, transport vectors, targets) with reference to five migration pathways:

- ground water,

- surface water, divided into two different migration components:

1. overland/flood migration component,

2. ground water to surface water migration component,

- air,

- direct contact inside the polluted site,

- direct contact outside the polluted site.

Each category includes a group of factor (or parameter). The values of these parameter are calculated using tables, normographs or plotted functions. The sum of the single scores define the global score of the investigated site. The score obtained with these methods are in the range from 0 to 100 [3].

On the other hand, Absolute Risk Analysis Methodologies like, for example, the US-EPA R.B.C.A. (Risk Based Corrective Action), require more detailed description of the sources, the transport pathways and the details of the pollutants absorption by the receptors, in terms of chemical/physical parameters and mechanisms. This description is performed via different kinds of simulation models, of semi-empirical and analytical nature or, in same cases, requiring very detailed computer numerical resolution algorithms.

In this work I would like to would focus my analysis on the aspect of the transport of the pollutant from the sources to the receptor sites and on the importance of the correct modelling of the pollutant fate. In particular, I am going to describe in detail a simulation model that we apply at the CRA, in conjunction with suitable models for the pollutants redistribution among phases at the source 


\section{Environmental Health Risk}

points and for the simulation of the effect of pollutants absorption by the receptors.

\section{Short description of a pollutant fate simulation models}

The model I would analyse in detail is an innovative simulation model for the pollutants transport in the underground water. This model has been initially developed for the simulation of the bioremediation process, both in the unsaturated zone as in the saturated one. Besides the application to the project and analysis of the bioremediation process this model can be usefully applied in the simulation of the pollutant fate and transport in the ground and in the underground water.

The model is based on the so called "Cellular Automata" (CA) paradigm [5], that allows a mathematical description of the chemical/physical phenomena in a different way with respect to the "more traditional" differential equation approach and related infinitesimal limit approximation for the equation numerical solution.

Let us recall that, in $\mathrm{CA}$, transition functions replace equations, as they describe the way how state variables change in time; however, in modelling macroscopic physical systems, it is often possible (as in our case) to distinguish between terms of the transition function which relate to intercellular processes, and those terms which describe processes which fully take place inside a cell. If we associate a CA cell to a macroscopic portion of the physical space (e.g.: a portion of soil), a classical example of intracellular process is that of chemical reactions, which take place between substances that are in the same cell (while, on the other hands, the transport of the chemicals trough the soil is an typical intercellular phenomenon).

In the case of intracellular processes, in our model we have preferred, where possible, to describe our algorithms in terms of differential, or difference, equations; this approach facilitates the mathematical description and simulation of the processes, and the simulation model implementation, too.

In the following paragraphs we shortly describe the main theoretical characteristics of the new mathematical model we have implemented [6].

\subsection{The chemicals fate modelling}

In polluted soils, chemicals can then be present in all the phases: as gas in soil air, as a dissolved chemical in the soil water, as dissolved chemical in the non aqueous phase, adsorbed to the soil organic matter. Consequentially, the total chemical concentration $C_{t}$ can be written as:

$C_{t}=\rho_{b} C_{a}+\theta C_{l}+\pi C_{n w}+a C_{g}$

where $C_{a}$ is the adsorbed chemical concentration (expressed as the mass of sorbant per mass of dry soil), $C_{l}$ the dissolved chemical concentration in the wetting phase (expressed as the mass of chemical per volume of wetting phase), $C_{n w}$ is the dissolved chemical concentration in the non wetting phase [NAPL] 
(expressed as the mass of chemical per mass of NAPL), $C_{g}$ is the gaseous chemical concentration (expressed as the mass of chemical vapour per volume of soil air). The units in which the chemical phases are expressed correspond to the way in which they are measured. For example, if a soil air sample of volume $V_{a}$ is withdrawn and a mass $M_{g}$ of chemical vapour is detected in it, then $C_{g}=M_{g} / V_{a}$. The soil density $\rho_{b}$, the NAPL density $\pi$, the volumetric water content $\theta$ (i.e. the volume of water per total volume) and the volumetric content $a$ convert each of these phase concentrations to mass per soil volume, which is the unit in which $C_{t}$ is expressed.

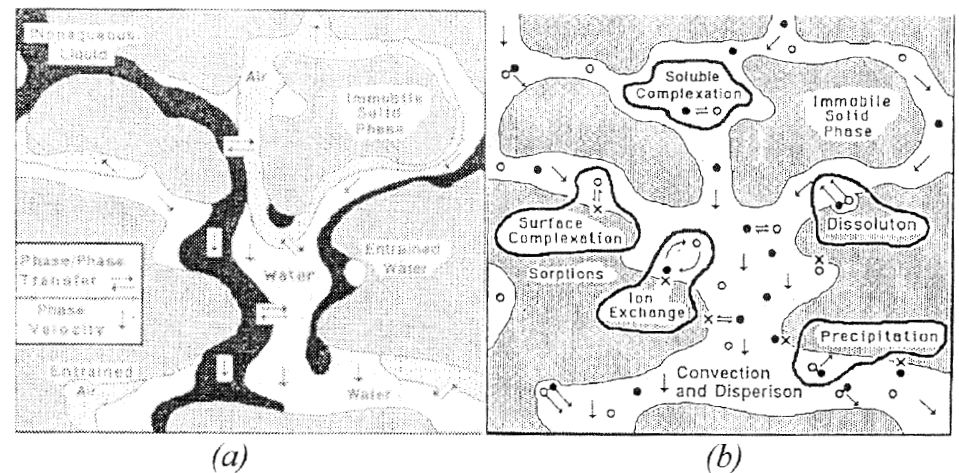

Figure 3: (a) Chemical flux among the phases; (b) Chemical phenomena.

The most relevant phenomena that can involve chemicals are:

- transport, that describes the motion of a chemical due to the motion of the phase in which it is present;

- molecular diffusion of a chemical within the phase that contains the chemical;

- flow of the chemical among phases (e.g. adsorption/desorption, transpiration);

- chemical reactions.

Transport and diffusion take place between different cells, while adsorption/ desorption and chemical reactions are supposed to occur inside each cell.

\subsection{The modelling of the mass transport}

We suppose that a chemical transported by a mobile phase mixes instantaneously with the chemical already present in the cell. The variation in the amount of chemical within the cell is given by the difference between the chemical that comes into the cell and the chemical that flows out from the cell. 
188 Environmental Health Risk

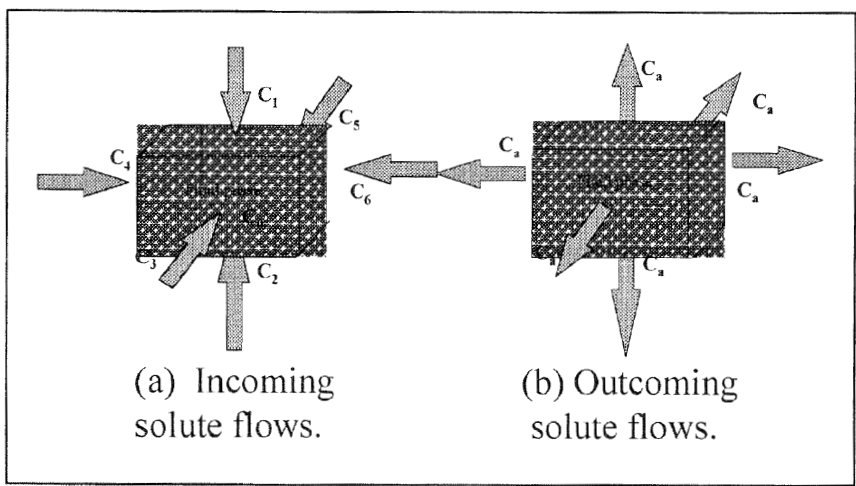

Figure 4: The incoming flows at time $\mathrm{T}$ carry different concentrations of chemical (in this example (from six different directions): the substance mixes immediately inside the cell, so that the outcoming flows (at time $\mathrm{T}+1$ ) carry the same concentration of solute.

The amount of chemical $Q_{C}$ ([I]) in the phase $\alpha$ that in a step of CA flows in (or out) the cell in the direction $i$ is:

$Q_{C}=J_{\alpha, i} \cdot C_{i}$

where $J_{\alpha, i}$ is the flow $\left(\left[1^{3} \mathrm{t}^{-1}\right]\right)$ of the phase $\alpha$ in the direction $i$ in the cell and $C_{i}$ is the concentration of the chemical in the cell $i$ of the neighbour $\left(\left[\mathrm{m} \mathrm{l}^{-3}\right]\right)$.

\subsection{The modelling of the mass diffusion}

This phenomenon takes place when there is a different concentration of achemical between neighbour cells of the CA.

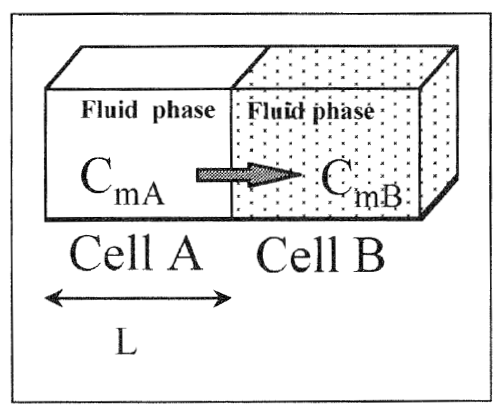

Figure 5: Representation of the diffusion flux between two neighbour cells. 
The amount of chemical in the phase $\alpha, Q_{C}([1])$, that diffuses in one step of the Cellular Automata is given by:

$$
Q_{C}=-D_{\alpha} \cdot \frac{\Delta C}{\Delta z} \cdot A
$$

where $D_{\alpha}$ is the diffusion coefficient $\left(\left[1^{2} \mathrm{t}^{-1}\right]\right)$ in the phase $\alpha$, and $C$ is the concentration of the chemical $C$ in the cell $\left(\left[\mathrm{m} \mathrm{l}^{-3}\right]\right)$ and $A\left(\left[\mathrm{l}^{2}\right]\right)$ is the area gone through from the chemical [3].

\subsection{Flow among phases, adsorption/desorption}

The phenomenon of "phase re-partition" takes place when a chemical moves from a phase to another phase; this kind of phenomenon is the first one that happens only inside a single cell. Flow among phases and adsorption/desorption (this is the name of the phase re-partition when one of the two involved phases is the soil) can be modelled in the same formal way.

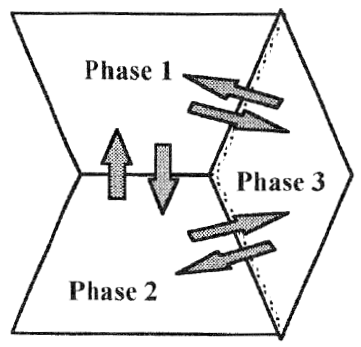

(a)

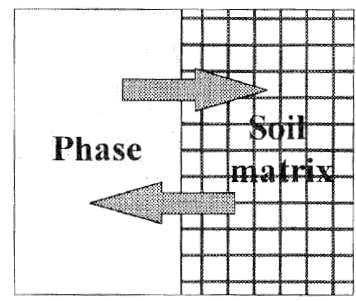

(b)

Figure 6: Flow among phases (a); adsorption/desorption (b).

The amount of the chemical $Q_{C}([\mathrm{~m}])$ that passes from phase $\alpha$ to phase $\mathrm{b}$ (in one step on the $\mathrm{CA}$ ) is given by:

$Q_{C}=Q_{o C} \exp \left(-\Gamma_{\alpha \rightarrow \beta} \cdot \Delta t\right)$

where $Q_{0 C}([\mathrm{~m}])$ is the amount of chemical $C$ at $t=t_{0}, \Gamma_{\alpha \rightarrow \beta}\left(\left[\mathrm{t}^{-1}\right]\right)$ is the exchange parameter among phase $\alpha$ and $\beta$ and $\Delta t$ is the time corresponding to one step on the CA. Notice that eq. (4) is directly the solution of a differential equation. This is indeed an advantage of using the cellular automata framework, where it is not necessary to numerically solve a lot of differential equations: when the solution is known and applicable to some cell, it can be directly incorporated into the transition function. 


\section{Environmental Health Risk}

\subsection{The modelling of the chemical reactions}

Typically, many different chemical reactions take place in soils: chemicals contained in a phase can react with other chemicals to form both soluble and/or insoluble (in a certain phase) products (it must be noted that products which are insoluble in water could be soluble in the NAPL phase and vice-versa); soluble species in water may also enter into oxidation/reduction, acid/base reactions, chelations, complexations, and several other types of association that result in soluble products. Almost it is quite practically impossible to know all the details about the interested reactions, Fortunately, on the other hand, it is frequently possible to individuate one or two slower steps which constitute the bottleneck of the reaction, so that the attention can be focused only upon these latter steps. In any case, all these kinds of interactions can be described using a common scheme, in which chemical species combine in quantities related to their stoichiometric coefficients and with a reaction rate that depends on many parameters, such as temperature, reagents concentration, the soil $\mathrm{pH}$ and so on. Each significant reaction occurring in the soil could be modelled by means of this framework; from the point of view of the cellular automaton, all these phenomena take place inside a single cell.

For example, the dissociation of hydrogen peroxide in oxygen and water:

$\mathrm{H}_{2} \mathrm{O}_{2} \rightarrow 1 / 2 \mathrm{O}_{2}+\mathrm{H}_{2} \mathrm{O}$

It has been modelled considering that the amount of oxygen produced by the dissociation $Q\left(\mathrm{O}_{2}\right)$ is related to the amount of hydrogen peroxide through their stoichiometric coefficients:

$Q\left(\mathrm{O}_{2}\right)=16 / 34 Q\left(\mathrm{H}_{2} \mathrm{O}_{2}\right)$

The kinetics of the $\mathrm{O}_{2}$ dissociation is modeled by means of a first order kinetics:

$$
C_{\mathrm{H}_{2} \mathrm{O}_{2}}(t+1)=C_{\mathrm{H}_{2} \mathrm{O}_{2}}(t) \cdot \exp (-\mu \cdot t)
$$

Therefore the corresponding variation of oxygen is given by:

$$
C_{O_{2}}(t+1)=C_{O_{2}}(t)+\Lambda_{O_{2}: H_{2} O_{2}} \cdot(1-\exp (-\mu \cdot t)) \cdot C_{H_{2} O_{2}}(t)
$$

\section{Some examples of applications}

Besides the possibility of application of this model to the project and analysis of the "in-situ" bioremediation intervention [1], it is possible to apply it for a detailed simulation of the contamination source shape and of the fate of the pollutant, typically organic hydrocarbon (Dense Non aqueous Phase LiquidsDNAPLs or Non aqueous Phase Liquids-NAPLs). In this way, it is possible to integrate the results of this simulation model with the procedures for the risk 
analysis evaluation, in order to obtain a more detailed estimation of the risk for the targets.

For example, at CRA we use this kind of model for the so called "Tier 3" of the R.B.C.A. methodology, where site specific numerical simulation models have to be applied for a detailed simulation of the forecasted pollutant fate in complex situation.

For example, I herewith present the results of the application of this model to a real field intervention, represented by a regular hexagon of about $20 \mathrm{~m}$ side. On each vertex and at the centre of the hexagon there is an extraction well. Inside each triangle which constitute the hexagon there are three injection wells; all these wells are working simultaneously following a timetable which produce a flow rate change each week. The water table is about at $9.0 \mathrm{~m}$. below ground level, and it is approximately $2.0 \mathrm{~m}$. deep; above the water table the soil is not saturated. No NAPL flow has been measured in the last year.

In our simulations we have supposed a constant hydraulic conductivity, and we used cubic cells having 2 meter of size. In order to complete the mass balance, we used suitable boundary condition cells. That is, the mass balance inside the field test is closed by simulating uniform water injection or extraction from the borders. The used schema containing the relevant simulation features is shown in the following figure 7 .

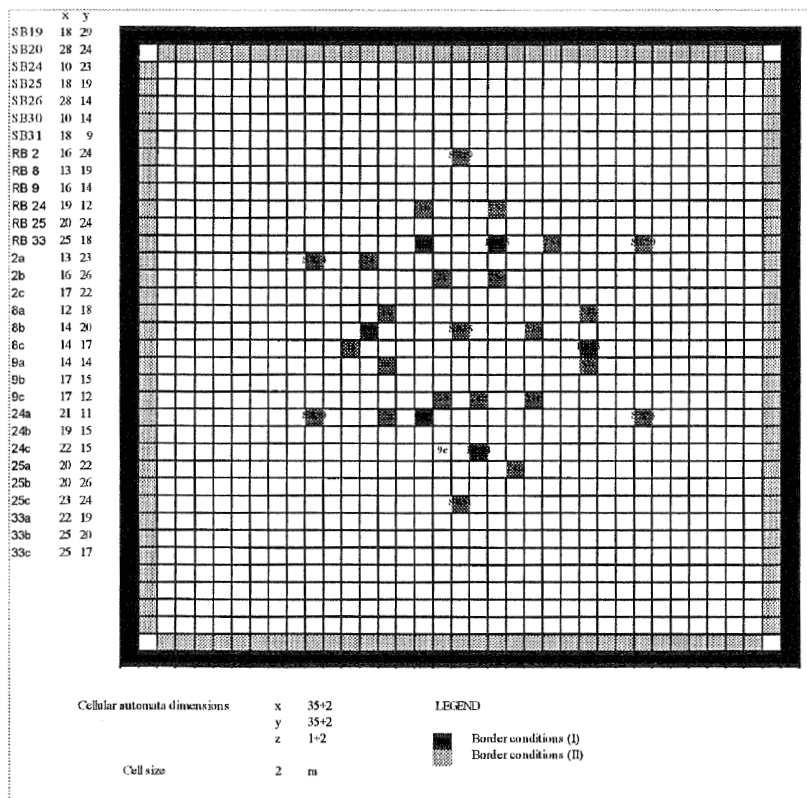

Figure 7: Cellular automata representation of field test

The model can run into two versions. One for single processor computers (under WINDOW operating system) and another one for parallel architectural computer (under UNIX). Typically, the mono-processor version of the model is 


\section{Environmental Health Risk}

suitable for the simulation of the saturated zone, when is required to simulate only two-phases fluxes (water and, in case, not dissolved pollutant). On the other hand, the multi-processor version of the model is generally required when you want to simulate the phenomena occurring in the vadose (unsaturated) portion of the soil, when three phases fluxes (water, not dissolved pollutant and air) have to be analysed.

The final results of the simulation of the water fluxes and of the contaminant profiles are reported in the figures 8 and 9. The simulations I herewith present have been done using a cluster of four Pentium III P.C., running under LINUX.

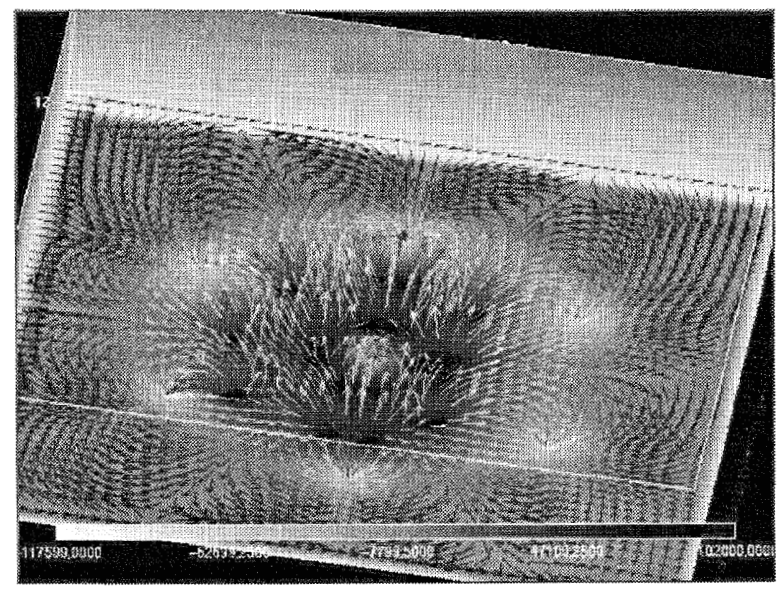

Figure 8: Horizontal section representation of the simulated water flux and pollutant concentration in a real case intervention

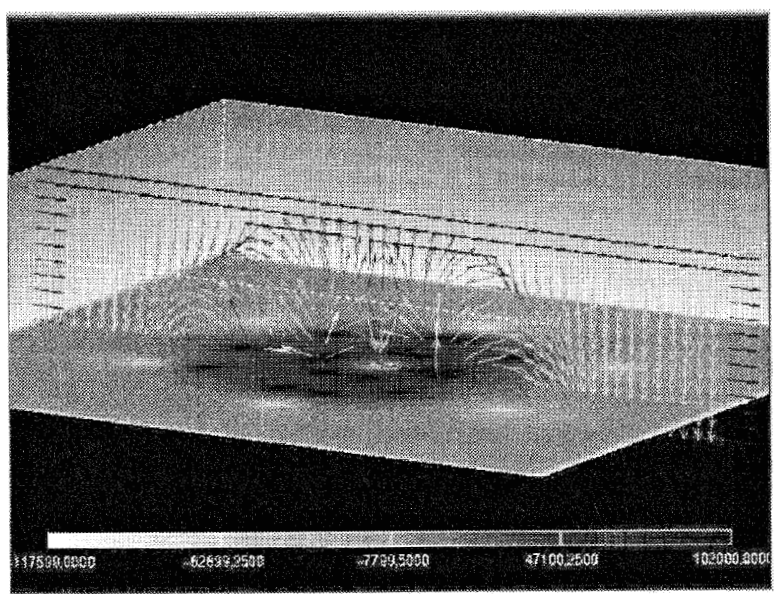

Figure 9: Vertical section representation of the simulated water flux and pollutant concentration in a real case intervention 


\section{Future research development}

As we can observe from the results reported in Figures 8 and 9, in many real world cases the water flow and contaminant patterns are very complicated. The standard approximations, normally applied in the risk analysis methods, may greatly underestimate the effect of the pollutant both on the source characterisation and on the transport of the contaminants. For these reasons at CRA we have started a research focused on the integration of the detailed Cellular Automata based model for the pollutant source shape and transport simulation with absolute risk analysis techniques.

If we refer to the general theoretical framework reported in figure 2 and to the Cellular Automata schemata, in this new approach, the global risk $R_{j}$ for the generic target $J$ can be expressed in the following way:

$R_{j}=\sum_{i} S_{i}(C) T_{i}\left(C, \bar{r}_{i j}\right) A_{j}(C)$

where:

- $\mathrm{S}_{\mathrm{i}}(\mathrm{C})$ is the source of contamination, due to the pollutant $\mathrm{C}$, estimated in the cell i of the Cellular Automata;

- $\mathrm{T}_{\mathrm{i}}\left(\mathrm{C}, \mathrm{r}_{\mathrm{ij}}\right)$ is the transport of the contaminant $\mathrm{C}$ from the source cell $\mathrm{i}$ to the receptor $\mathrm{j}$, that depends on the relative distances $\mathrm{r}_{\mathrm{ij}}$;

- $A_{j}(C)$ is the contaminant $C$ adsorption by the target $j$.

Naturally, $T_{i}\left(C, r_{i j}\right)$ and $S_{i}(C)$ can be estimated via simulation models of the kind we have previously shown. These new and innovative approach will allow, in the next future, an easy methodology for a more precise estimation of the risk connected with polluted site.

\section{Acknowledgement}

The author wishes to thank Dr. Marco Villani and Dr. Roberto Serra for their contribution to the COLOMBO Project. A particular thank to Miss Susanna Nagj for her help and check of the spelling of the paper.

\section{References}

[1] Andretta M., et al. Bioremediation modelling: from the pilot plants to the field, Innovative Methods in Support of Bioremediation, Battelle Press: Columbus and Richland, pp 131-138, 2001.

[2] Andretta M., Cagnoli P., Elisee C., Favero V. Two new methodologies for the hazard ranking of polluted sites: the CHARSY (C.r.a Hazard Ranking System) and the RER methods, Risk Analysis, WITPress, Computational Mechanics Publications: Southampton and Boston, pp.103-112, 1998. 


\section{Environmental Health Risk}

[3] Andretta M., et al. Comparison among polluted soils hazard ranking systems and absolute risk analysis methodologies, Risk Analysis II, WIT Press, Southampton and Boston, pp.456-463, 2000.

[5] Serra R., Di Gregorio S., Villani M., Andretta M. Bioremediation simulation models, Biotechnology for soil remediation, ed. Serra R., Cipa Editore, Milano, 1998.

[6] Di Gregorio S., Serra R., Villani M. A Cellular Automata model of soil bioremediation, Complex systems, 11(1), pp. 31-54, 1997. 